\title{
Observation of Fowler-Nordheim hole tunneling across an electron tunnel junction due to total symmetry filtering
}

\author{
M. Bowen, ${ }^{1, *}$ A. Barthélémy, ${ }^{1}$ V. Bellini, ${ }^{2,3}$ M. Bibes,${ }^{1, \dagger}$ P. Seneor, ${ }^{1}$ E. Jacquet, ${ }^{1}$ J.-P. Contour, ${ }^{1}$ and P. H. Dederichs ${ }^{2}$ \\ ${ }^{1}$ Unité Mixte de Physique CNRS/Thales, Route Départementale 128, 91767 Palaiseau Cedex, France \\ and Université Paris-Sud XI, 91405 Orsay Cedex, France \\ ${ }^{2}$ Institut für Festkörperforschung, Forschungszentrum Jülich, D-52425 Jülich, Germany \\ ${ }^{3}$ INFM-National Research Center on nanoStructures and bioSystems at Surfaces (S3) and Dipartimento di Fisica, \\ Università di Modena e Reggio Emilia, Via Campi 213/A, I-41100 Modena, Italy
}

(Received 24 August 2005; revised manuscript received 9 March 2006; published 27 April 2006)

\begin{abstract}
Using a $\mathrm{SrTiO}_{3}(001)$ barrier and $\mathrm{La}_{0.7} \mathrm{Sr}_{0.3} \mathrm{MnO}_{3}(001)$ electrodes, we study the case of coherent spinpolarized tunneling of electrons that exhibit a symmetry mismatch with respect to the lower electron barrier height. Due to this mismatch, electrons with energy above this barrier height continue to tunnel thanks to total symmetry filtering. This allows us to observe symmetry-matched hole tunneling across this electron tunnel junction, and in particular spin-polarized oscillations in the Fowler-Nordheim hole regime above the hole barrier height, thereby enriching the present theoretical picture of coherent spin-polarized tunneling at finite bias.
\end{abstract}

DOI: 10.1103/PhysRevB.73.140408

PACS number(s): 75.47.Lx, 72.25.Mk, 73.40.Gk, 85.75.-d

The experimental study of spin conservation in the course of the solid-state tunneling process of charge carriers was initially performed across amorphous barriers using polycristalline electrodes. ${ }^{1,2}$ A ferromagnetic electrode with differing densities of states (DOSs) at the Fermi level $E_{F}$ for the two spin populations is used to achieve the spin polarization of the current, which may be detected thanks to either a superconducting or a ferromagnetic counterelectrode. The latter system is a magnetic tunnel junction (MTJ).

To further understand this fundamental transport process, one may investigate the conservation of other carrier properties such as momentum and the wave-function symmetry, thereby requiring fully epitaxial MTJs. This coherence in spin-polarized tunneling has been fruitfully studied over the past four years in the particular case of the $\mathrm{Fe} / \mathrm{MgO}(001)$ system thanks to a convergence between theory ${ }^{3,4}$ and experiment. ${ }^{5-8}$ Since the $\mathrm{MgO}(001)$ tunnel barrier filters charge carriers with $\Delta_{1}$ electronic symmetry that are fully spin-polarized at $E_{F}$ for $\mathrm{Fe}(001)$, this matching of electronic symmetries between electrodes and barrier in this system should lead to very large values of tunneling magnetoresistance (TMR) that currently reach $300 \%$ experimentally. ${ }^{6-8}$ Within the Julliere model, ${ }^{9}$ the enhanced spin polarization due to this symmetry filtering effect reaches $P=77 \%$, compared to $P=45 \%$ without such coherent tunneling. ${ }^{10}$

In noncoherent tunneling, the barrier height $\Phi$ that charge carriers perceive is the energy difference between $E_{F}$ at the junction interface and the nearest band edge of the barrier. Once $\Phi$ is overcome with a bias $V$ applied across the tunnel barrier, the tunneling process enters the Fowler-Nordheim (FN) regime, ${ }^{11}$ in which electrons now find available states in the barrier. This decrease in the perceived insulating thickness of the barrier leads ${ }^{12}$ to a large increase in tunneling conductance.

Taking into account coherence, electrons (holes) tunnel with respect to empty (filled) bands with the same electronic symmetry. In the case of the $\mathrm{Fe} / \mathrm{MgO}(001)$ system, this cor- responds to the lowest $\Phi$ as in the noncoherent case. Unfortunately, for $e V>\Phi$, the effective short circuit of the junction does not allow one to then probe tunneling with respect to other bands in the barrier. As such, the case of symmetry matching between tunneling carriers and states that define the lowest barrier height is fundamentally very limiting. Moreover, it is quite puzzling that no hallmark features of FN tunneling have been reported thus far in the $\mathrm{Fe} / \mathrm{MgO}$ system. This experimental shortcoming is compounded by the difficult challenge of theoretically describing coherent tunneling at finite bias ${ }^{13}$ to account for experiment (see, e.g., Ref. 5). Achieving an in-depth understanding of coherent spin-polarized tunneling for $e V>\Phi$ remains of fundamental importance. Indeed, the coherent FN processes that occur within the barrier will eventually be described through a merging of tunneling magnetoresistance and giant magnetoresistance (GMR) (Ref. 14) physics across insulating and metallic spacers, respectively.

The experiments and theory contained here seek to explore this aspect of spin-polarized coherent tunneling. We describe the more general case of coherent tunneling of electrons that exhibit a symmetry mismatch with respect to the lowest barrier height of the transition metal oxide $\mathrm{SrTiO}_{3}$ (STO). For an applied bias which exceeds this electron barrier height, although the STO barrier exhibits empty states of the conduction band (CB) at that energy, we observe enduring tunneling due to this symmetry mismatch. We argue that it is only when the applied bias overcomes the barrier height defined by the band with the same symmetry-the valence band (VB) here-that junction current rises dramatically. This case allows us to study the regime of FN hole tunneling across this electron MTJ.

To contrast $\mathrm{MgO}(001)$ and $\mathrm{STO}(001)$ when used as tunnel barriers, we present in Fig. 1 their complex band structures. ${ }^{15}$ In these density-functional theory local-density approximation (DFT-LDA) calculations, ${ }^{16}$ performed within the screened KKR framework, ${ }^{17,18}$ the experimental lattice con- 

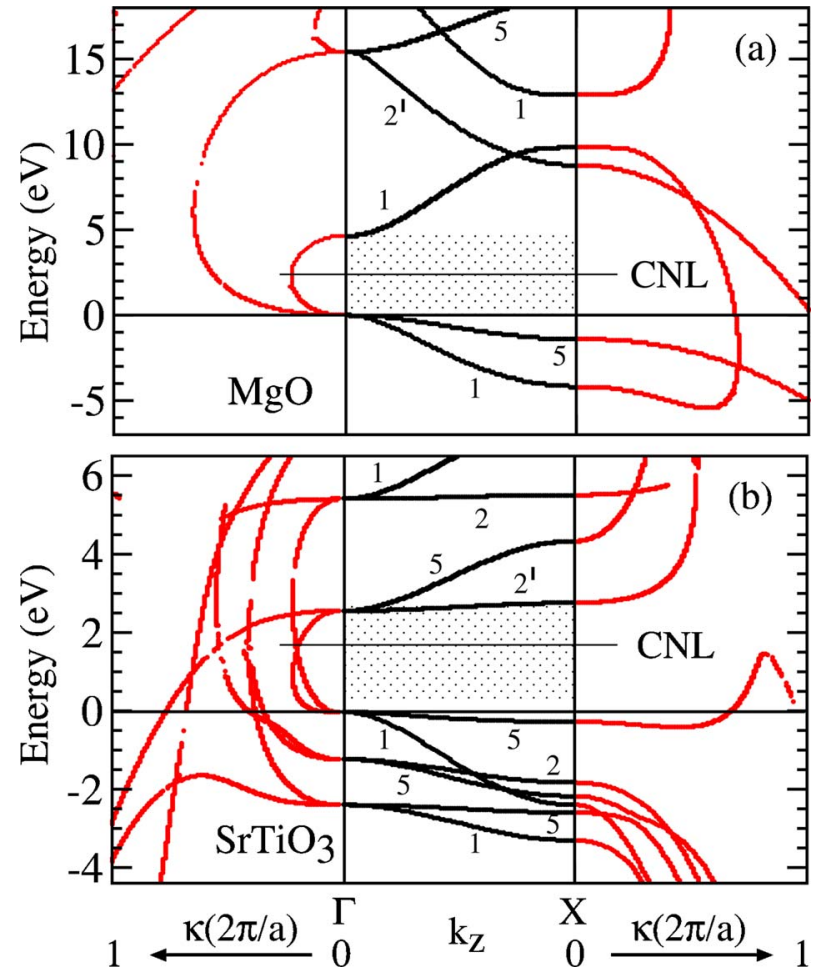

FIG. 1. (Color online) Complex band structures of (a) $\mathrm{MgO}$ and (b) $\mathrm{SrTiO}_{3}$ along [001]. Numbers indicate the $\Delta$ symmetries (e.g., $\left.1=\Delta_{1}\right)$. The CNL is the charge neutrality level.

stants $a_{\mathrm{MgO}}=4.2 \AA$ and $a_{\mathrm{STO}}=3.905 \AA$ for $\mathrm{MgO}$ and $\mathrm{STO}$ were used. To determine the tunnel barrier profile, we examine the complex bands across the direct band gap for $\mathrm{MgO}(001)$ and $\mathrm{STO}(001)$, i.e., at the $\Gamma$ point. ${ }^{19}$ The charge neutrality level (CNL) pinpoints the energy level within the band gap with equal decay lengths $\kappa$ of metal-induced gap states from the VB and $\mathrm{CB}$ which promote electron and hole tunneling, respectively. In conventional oxides with the same symmetry for the CB and $\mathrm{VB}$ (for $\mathrm{MgO}$ it is $\Delta_{1}$ ), only one $\kappa$ loop symmetrically connects these bands across the band gap. In this case, the CNL trivially lies in the center of the gap [see Fig. 1(a)]. In contrast, the VB and $\mathrm{CB}$ of a transition-metal oxide generally exhibit different symmetries. In the case of STO, the VB has $\Delta_{1}$ and $\Delta_{5}$ symmetries, while the CB has $\Delta_{2}$, and $\Delta_{5}$ symmetries [see Fig. 1(b)]. This results in several $\kappa$ loops across the direct band gap, such that in the case of STO the CNL is calculated to lie closer to the $\mathrm{CB}$. When in contact with a metal, $E_{F}$ at the interface will lie between the chemical potential of the insulator-which in the case of $\mathrm{STO}(001)$ is closer to the $\mathrm{CB}$ for both intrinsic and light $p$ or $n$ doping ${ }^{20,21}$ - and the CNL depending on the latter's pinning strength. ${ }^{22}$ From this discussion we infer that $E_{F}$ in our STO-based junctions lies closer to the $\mathrm{CB}$, such that the electron barrier height $\Phi_{e}$ with $\Delta_{2^{\prime}}$ and $\Delta_{5}$ symmetries is lower than the hole barrier height $\Phi_{h}$ with $\Delta_{1}$ and $\Delta_{5}$ symmetries, thereby defining an electron tunnel junction.

As our source of tunneling carriers, we use $\mathrm{La}_{0.7} \mathrm{Sr}_{0.3} \mathrm{MnO}_{3}(001)$ (LSMO) electrodes. In such fully epitaxial LSMO/STO/LSMO MTJs, the tunneling current consists of almost only spin-up carriers ${ }^{23,24}$ that in addition have

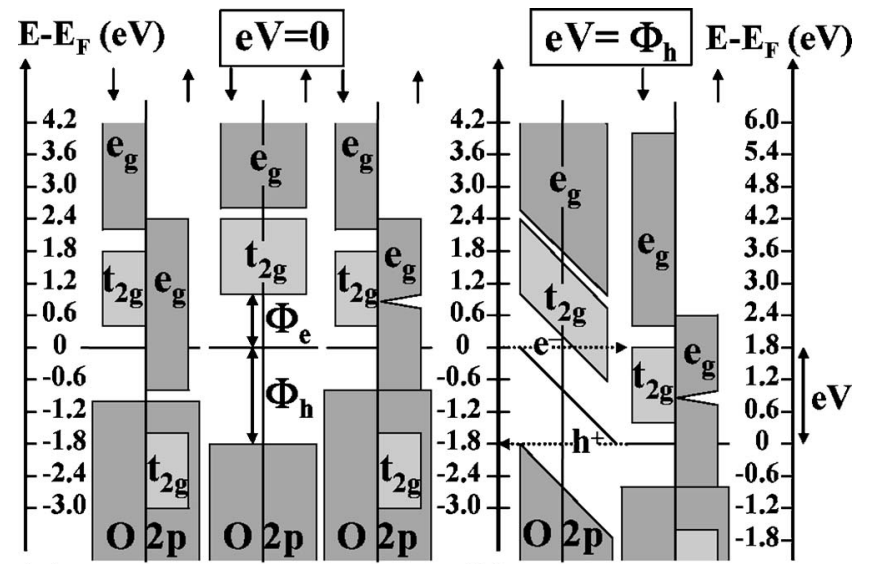

(a) LSMO STO LSMO (b) STO LSMO

FIG. 2. Schematic of the electronic symmetry resolved DOS of $\mathrm{STO}(001)$ and the LSMO/STO(001) interfaces for (a) $e V=0$ and (b) $e V=\Phi_{h}$. Here $\Delta_{2^{\prime}, 5}$ is $t_{2 g}$ and $\Delta_{1}$ is $\left(e_{g}, \mathrm{O} 2 p\right)$. In part from Refs. 20,21 , and 25-27.

only the $\Delta_{1}$ electronic symmetry. No in-plane rotation of the crystallographic axes occurs between $\operatorname{LSMO}(001)$ and STO(001) during growth. We thus directly compare the electronic symmetries between $\operatorname{LSMO}(001)$ and $\mathrm{STO}(001)$ across such MTJs. Starting from bulk data culled from Refs. 20, 21, and 25-27 and consistent with what follows, we present in Fig. 2(a) a schematic of the electronic symmetryresolved DOS of our ultrathin $\mathrm{STO}(001)$ barrier and the $\mathrm{LSMO} / \mathrm{STO}(001)$ interfaces at $V=0$ to illustrate the resulting symmetry mismatch between LSMO conduction carriers and the STO electron barrier height of lowest energy here. The coherent tunneling of LSMO $\Delta_{1}$ carriers should proceed with respect to the bands with the correct $\Delta_{1}$ symmetry that are linked at the $\Gamma$ point by a complex loop [see Fig. 1(b)]. As argued above, the interfacial $E_{F}$ lies between the STO CB edge and the CNL. Since this complex loop is rather flat within this energy range, both electron and hole tunneling with $\Delta_{1}$ symmetry may occur. This fact is valid at the $\Gamma$ point, and may therefore apply only to a low-bias regime. ${ }^{13}$

We now present our experimental results. STO(001)// $\operatorname{LSMO}(350 \AA) / \operatorname{STO}(27.8 \AA) / \mathrm{LSMO}(100 \AA)$ trilayers were grown by the pulsed laser deposition of LSMO and STO. Samples were then patterned into junctions using UV lithography, ${ }^{23}$ and transport data measured in four-point geometry using a dc-voltage source. Figure 3 presents the response with bias $\Delta V$ applied between the lower and upper LSMO electrodes of a $2 \times 6 \mu \mathrm{m}^{2}$ junction at $T=10 \mathrm{~K}$. Panel (a) shows an overview of TMR $=\left(I_{\mathrm{P}}-I_{\mathrm{AP}}\right) / I_{\mathrm{AP}}$ and differential TMR, diff. TMR $=\left(G_{\mathrm{P}}-G_{\mathrm{AP}}\right) / G_{\mathrm{AP}}$, using current $I$ and differential conductance $G=d I / d V$ (hereafter called conductance) data in the parallel (P) and antiparallel (AP) orientations of the electrodes' magnetization. Thus defined, both TMR and diff. TMR may reach $+\infty$ or $-100 \%$. The relatively low TMR value at $V \sim 0$ reflects the slight imperfection of one interface as discussed below. For a finite bias below the junction's lowest barrier height, the spectroscopic nature of tunneling governs the junction's bias-dependent response in the absence of inelastic processes. Here, past the spin-wave excitation regime spanning $\sim 150 \mathrm{mV}$, the TMR curve ex- 


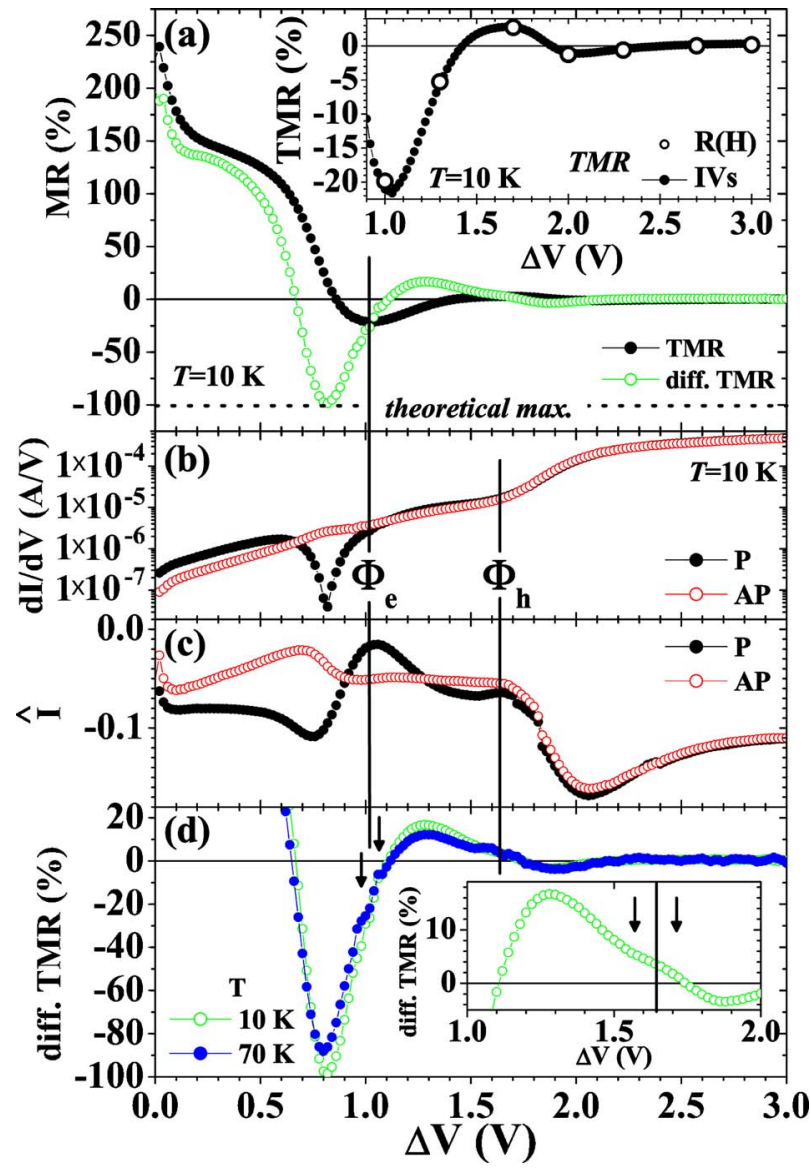

FIG. 3. (Color online) Bias dependencies of (a) TMR and diff. TMR, (b) differential conductance, and (c) $\hat{\mathrm{I}}(10 \mathrm{~K}, 70 \mathrm{~K})$ $=[I(70 \mathrm{~K})-I(10 \mathrm{~K})] / I(10 \mathrm{~K})$ in the parallel $(\mathrm{P})$ and antiparallel (AP) states. Panel (d) and its inset, and the inset to panel (a), provide closeups of diff. TMR and TMR, respectively.

hibits a plateau, followed by a renewed decrease once the applied bias exceeds the LSMO minority gap $\delta=350 \mathrm{meV}$ defined by a $t_{2 g}\left(\Delta_{2}^{\prime}\right)$ band. ${ }^{25}$ This means that $\Delta_{1}$ electrons tunnel coherently across the barrier into the collecting electrode, and eventually reach $\Delta_{2}$, states away from the interface through a decoherence process in this AP conduction channel, similarly to the case of $\mathrm{Fe} / \mathrm{MgO}(001)$ (Ref. 3).

Figure 3(b) shows the bias dependencies of $G_{\mathrm{P}}$ and $G_{\mathrm{AP}}$. At $V_{\mathrm{g}}=+0.82 \mathrm{~V}$, the parallel conductance $G_{\mathrm{P}}$ drops to $G_{\mathrm{P}}\left(V_{g}\right) \ll G_{P}(V=0)$ while $G_{\mathrm{AP}}$ does not. This reflects the unsuccessful injection of fully spin $\uparrow$-polarized electrons from the top electrode at $E_{F}$ toward the atomically distorted lower electrode interface and into a pseudogap at $E=E_{F}+e V_{g}$ in the $e_{g}^{\uparrow}$ manifold. ${ }^{24}$ Referring to Fig. 2, we note in passing how a similar analysis in terms of hole conduction for this $\mathrm{P}$ coherent tunneling channel may be carried out, though only qualitatively at this time due to the difficulty in self-consistently establishing the LSMO DOSs far from $E_{F}$ at both interfaces based on bulk data. While TMR $\sim I$, diff. TMR $\sim G$ and therefore does not convolute the electrode DOSs and the barrier transmission matrix with applied bias. ${ }^{12}$ Indeed, diff. TMR reaches $-99 \%$ at exactly $V_{g}$, so that it is more pertinent here to study bias-dependent features using diff. TMR.

We now discuss the junction potential profile. On the logarithmic scale of Fig. 3(b), the large rise in both $G_{\mathrm{P}}$ and $G_{\mathrm{AP}}$ reflects exceeding a barrier height $\Phi=1.65 \mathrm{eV}$. A similar value was found for $\Delta V<0$. We confirm $\Phi$ by examining $\hat{\mathrm{I}}(10 \mathrm{~K}, 70 \mathrm{~K})=I[(T=70 \mathrm{~K})-I(T=10 \mathrm{~K})] / I(T=10 \mathrm{~K}) \quad[\mathrm{see}$ Fig. 3(c)]. Indeed, Î exhibits a peak at the same bias value as well as a similar bias dependence above this value for both the $\mathrm{P}$ and AP configurations. Such peaks reflect the additional tunneling current due to carriers injected at the barrier height with additional energy $k_{\mathrm{B}} T$ (Refs. 28 and 29).

Since the bulk STO band gap is $E_{g}=3.2 \mathrm{eV}$ (Ref. 30), and is reduced in ultrathin films, ${ }^{31}$ the striking observation is that $\Phi>E_{g} / 2$. Since $\Phi_{e}<E_{g} / 2$ as seen in Fig. 2(a), this implies that $\Phi$ is not $\Phi_{e}$, with two consequences. (i) Aside from DOS-related effects, the same rate of conductance increase with no abrupt change for $e V<\Phi$ implies that, once $e V$ $>\Phi_{e}, \Delta_{1}$ electrons continue to tunnel, despite the presence in STO of empty states with $\left(\Delta_{2^{\prime}}, \Delta_{5}\right)$ symmetry at that energy [see Fig. 2(b)], due to symmetry filtering. (ii) $\Phi$ reflects the energy difference between $E_{F}$ and the nearest $\Delta_{1}$ band edge, i.e., $\Phi=\Phi_{h}$ [see Fig. 2(a)]. This means that we observe hole tunneling across this electron tunnel junction.

Given the efficiency of symmetry filtering above $\Phi_{e}$, experimental indications of $\Phi_{e}$ are more indirect. Expecting a loss of ideality of our tunneling current with increasing $T$, we again examine $\hat{\mathrm{I}}$ for signatures of $\Phi_{e}$. Aside from DOSrelated effects due to the pseudogap at $E-E_{F}=0.82 \mathrm{eV}$, we find small peaks in the $\mathrm{P}$ and $\mathrm{AP}$ data of $\hat{\mathrm{I}}$ at $V \simeq+1 \mathrm{~V}$ [see Fig. 3(c)] that could be related to $\Phi_{e}$. Supposing that $\Phi_{e}$ $=1 \mathrm{eV}, E_{g}=\Phi_{e}+\Phi_{h}=2.65 \mathrm{eV}$ here [see Fig. 2(a)], which for this ultrathin ${ }^{31}$ layer compares favorably to the $3.2-\mathrm{eV}$ bulk value.

Referring to Fig. 2(b), the $\mathrm{FN}$ regime (i.e., when $\mathrm{eV}$ $>\Phi_{h}$ here) reflects how electrons from the fully occupied VB of STO tunnel with respect to this band edge toward $E_{F}$ of the right-hand electrode, amounting to hole transport. In this regime, we observe several oscillations in $\operatorname{TMR}(V)$ [see inset of Fig. 3(a)], which were confirmed by $R(H)$ sweeps at discrete bias values. ${ }^{32}$ More precisely, the onset of the FN regime at $e V \simeq \Phi_{h}$ is associated with kinks in diff. TMR [see arrows in the inset of Fig. 3(d)]. Such kinks reflect the change in the reflection/transmission coefficients of the tunneling carrier at the collecting interface. The presence of a set of kinks could reflect the sharpness of the interface energy profile. In line with our above discussion, we also observe kinks in diff. TMR for $T=70 \mathrm{~K}$ at $e V \simeq \Phi_{e}$ [see arrows in Fig. 3(d)] that are hardly resolved at $T=10 \mathrm{~K}$. Thus, the diff. TMR data allow us to corroborate $\Phi_{e}$ and $\Phi_{h}$.

Oscillations in diff. TMR beyond these kinks are also observed. This is due to quantum well states perceived by carriers in the coherent FN regime across the STO spacer. Indeed, coherent FN tunneling embodies the transport of carriers at an energy across the electric-field-distorted spacer corresponding to an insulating portion and a portion with states of the same electronic symmetry that is therefore perceived as metallic [see Fig. 2(b)]. Coherent transport processes through these two portions reflect spanning vectors across the complex and real bands at that energy, respectively. This is similar to theory developed at the Fermi level to extend giant magnetoresistance, ${ }^{33}$ and we note how the Fermi surface of our STO spacer supports spanning vectors 
for both charge carriers. ${ }^{30}$ As such, coherent FN tunneling represents the intersection between the physics of GMR and TMR, and in this sense may be compared to tunneling into a spacer layer inserted at the junction interface as studied by Yuasa et al $^{34}$ In their case, the metallic spacer thickness may be varied during sample growth, and the spanning vector modified with applied bias. In coherent FN tunneling both the metallic spacer thickness and the spanning vector vary with applied bias.

In conclusion, we have presented a picture of coherent tunneling across the $\mathrm{SrTiO}_{3}$ barrier of a magnetic tunnel junction that involves a symmetry mismatch between the wave function of conduction carriers and the lowest barrier height. Using this more general case, we provided this description of both electron and hole transport across a MTJ. Our work should foster further theory on coherent tunneling at finite bias, in particular in the coherent Fowler-Nordheim regime. Studies of symmetry-polarized tunneling across both the insulating barrier and a suitably chosen metallic interfacial layer should enhance our picture of coherent tunneling and offer ideas for spintronic devices. Finally, we have shown how coherent tunneling can lead to an effective increase in the insulator's gap, with the immediate important application of reducing the leakage current in an epitaxial transistor.

We thank P. Mavropoulos, P. Levy, and H. Grahn for stimulating discussions. M.B. gratefully thanks the French government for financing. We acknowledge the European RTN on "Computational Magnetoelectronics" for partial support and a fruitful scientific environment.
*Email address: martin.bowen@ipcms.u-strasbg.fr. Present address: IPCMS, 23 rue du Loess, 67037 Strasbourg, France.

†Present address: Institut d'Electronique Fondamentale, Bât. 220, Université Paris-Sud, 91405 Orsay, France.

${ }^{1}$ R. Meservey and P. Tedrow, Phys. Rep. 238, 173 (1994).

${ }^{2}$ J. S. Moodera, L. R. Kinder, T. M. Wong, and R. Meservey, Phys. Rev. Lett. 74, 3273 (1995).

${ }^{3}$ W. H. Butler, X. G. Zhang, T. C. Schulthess, and J. M. MacLaren, Phys. Rev. B 63, 054416 (2001).

${ }^{4}$ J. Mathon and A. Umerski, Phys. Rev. B 63, 220403(R) (2001).

${ }^{5}$ M. Bowen, V. Cros, F. Petroff, A. Fert, C. M. Boubeta, J. CostaKrämer, J. Anguita, A. Cebollada, F. Briones, J. M. de Teresa et al., Appl. Phys. Lett. 79, 1655 (2001).

${ }^{6}$ J. Faure-Vincent, C. Tiusan, E. Jouguelet, F. Canet, M. Sajieddine, C. Bellouard, E. Popova, M. Hehn, F. Montaigne, and A. Schuhl, Appl. Phys. Lett. 82, 4507 (2003).

${ }^{7}$ S. Yuasa, T. Nagahama, A. Fukushima, Y. Suzuki, and K. Ando, Nat. Mater. 3, 868 (2004)

${ }^{8}$ S. Parkin, C. Kaiser, A. Panchila, P. M. Rice, B. Hughes, M. Samant, and S. Yang, Nat. Mater. 3, 862 (2004).

${ }^{9}$ M. Jullière, Phys. Lett. 54A, 225 (1975).

${ }^{10}$ D. Monsma and S. Parkin, Appl. Phys. Lett. 77, 720 (2000).

${ }^{11}$ R. Fowler and L. Nordheim, Proc. R. Soc. London 119, 173 (1928).

${ }^{12}$ J. Bardeen, Phys. Rev. Lett. 6, 57 (1961).

${ }^{13}$ C. Zhang, X.-G. Zhang, P. S. Krstić, H. P. Cheng, W. H. Butler, and J. M. MacLaren, Phys. Rev. B 69, 134406 (2004).

${ }^{14}$ M. N. Baibich, J. M. Broto, A. Fert, F. Nguyen Van Dau, F. Petroff, P. Etienne, G. Creuzet, A. Friederich, and J. Chazelas, Phys. Rev. Lett. 61, 2472 (1988).

${ }^{15}$ P. Mavropoulos, N. Papanikolaou, and P. H. Dederichs, Phys. Rev. Lett. 85, 1088 (2000).

${ }^{16}$ V. Bellini, Ph.D. thesis, Rheinisch-Westfälischen Technischen Hochschule Aachen, 2000.

${ }^{17}$ R. Zeller, P. H. Dederichs, B. Ujfalussy, L. Szunyogh, and P. Weinberger, Phys. Rev. B 52, 8807 (1995).
${ }^{18}$ K. Wildberger, R. Zeller, and P. H. Dederichs, Phys. Rev. B 55, 10074 (1997).

${ }^{19}$ At $V=0, k_{\|}$points along $\Gamma$-M contribute equally to $\Delta_{1}$ tunneling across STO(001), see Phys. Rev. Lett. 95, 216601 (2005). Similar calculations at large bias are lacking, so we consider the $\Gamma$ point where the tunnel barriers are lowest.

${ }^{20}$ T. Higuchi, T. Tsukamoto, N. Sata, M. Ishigame, Y. Tezuka, and S. Shin, Phys. Rev. B 57, 6978 (1998).

${ }^{21}$ T. Higuchi, S. Nozawa, T. Tsukamoto, H. Ishii, R. Eguchi, Y. Tezuka, S. Yamaguchi, K. Kanai, and S. Shin, Phys. Rev. B 66, 153105 (2002).

${ }^{22}$ J. Tersoff, Phys. Rev. Lett. 52, 465 (1984).

${ }^{23}$ M. Bowen, M. Bibes, A. Barthélémy, J.-P. Contour, A. Anane, Y. Lemaître, and A. Fert, Appl. Phys. Lett. 82, 233 (2003).

${ }^{24}$ M. Bowen, A. Barthélémy, M. Bibes, E. Jacquet, J.-P. Contour, A. Fert, D. Wortmann, and S. Blügel, J. Phys.: Condens. Matter 17, L407 (2005).

${ }^{25}$ M. Bowen, A. Barthélémy, M. Bibes, E. Jacquet, J.-P. Contour, A. Fert, F. Ciccacci, L. Duò, and R. Bertacco, Phys. Rev. Lett. 95, 137203 (2005).

${ }^{26}$ J.-H. Park, E. Vescovo, H.-J. Kim, C. Kwon, R. Ramesh, and T. Venkatesan, Nature (London) 392, 794 (1998).

${ }^{27}$ M. Zhuang, W. Zhang, and N. Ming, Phys. Rev. B 56, 14547 (1997).

${ }^{28}$ K.-H. Gundlach, J. Appl. Phys. 44, 5006 (1973).

${ }^{29}$ The use of free-electron models in a low-bias approximation is inappropriate for this system.

${ }^{30}$ Y. Aiura, I. Hase, H. Bando, T. Yasue, T. Saitoh, and D. S. Dessau, Surf. Sci. 515, 61 (2002).

${ }^{31}$ A. Ohtomo, D. Müller, J. Grazul, and H. Hwang, Nature (London) 419, 378 (2002).

${ }^{32}$ At $V=3 \mathrm{~V}, R=8 \mathrm{k} \Omega \gg R_{\text {Leads }}$, so $T M R=+0.2 \%$ is accurate. See R. van de Veerdonk, J. Nowak, R. Meservey, J. Moodera, and W. de Jonge, Appl. Phys. Lett. 71, 2839 (1997).

${ }^{33}$ P. Bruno, Phys. Rev. B 52, 411 (1995).

${ }^{34}$ S. Yuasa, T. Nagahama, and Y. Suzuki, Science 297, 234 (2002). 